Meta

Journal des traducteurs

Translators' Journal

\title{
Les entreprises commerciales
}

\section{Wallace Schwab}

Volume 24, numéro 1, mars 1979

La traduction juridique

URI : https://id.erudit.org/iderudit/004064ar

DOI : https://doi.org/10.7202/004064ar

Aller au sommaire du numéro

Éditeur(s)

Les Presses de l'Université de Montréal

ISSN

0026-0452 (imprimé)

1492-1421 (numérique)

Découvrir la revue

Citer cet article

Schwab, W. (1979). Les entreprises commerciales. Meta, 24(1), 177-212.

https://doi.org/10.7202/004064ar

Ce document est protégé par la loi sur le droit d'auteur. L'utilisation des services d'Érudit (y compris la reproduction) est assujettie à sa politique d'utilisation que vous pouvez consulter en ligne.

https://apropos.erudit.org/fr/usagers/politique-dutilisation/
Cet article est diffusé et préservé par Érudit.

Érudit est un consortium interuniversitaire sans but lucratif composé de l’Université de Montréal, l'Université Laval et l'Université du Québec à Montréal. Il a pour mission la promotion et la valorisation de la recherche. https://www.erudit.org/fr/ 


\section{Les entreprises commerciales}

Au cours du $x x^{e}$ siècle, les entreprises occidentales ont connu un tel accroissement d'activité économique qu'il a fallu adapter les structures à des besoins en évolution continuelle. Des pays d'Europe et d'Amérique, il en résulte aujourd'hui nombre de formes d'entreprises dont on se perd à tenter de comparer les ressemblances ou de mettre en relief les différences. Parfois, entre pays voisins, les institutions présentent des structures à ce point semblables qu'on est enclin à les confondre. Tel est le cas, par exemple, de la « société en nom collectif », connue sous ce nom en Belgique, en France et au Québec. En Allemagne, elle s'appelle Personengesellschaft, et Sociedad en nombre colectivo en Amérique latine. Enfin, on l'appelle Partnership en Angleterre, aux États-Unis et au Canada.

Lorsqu'on considère d'autres cas, il s'agit d'emprunts entre droits nationaux distincts, tels la Gesellschaft mit beschränkter Haftung d'Allemagne, dont la France s'est inspirée en 1925 pour créer la Société à responsabilité limitée, ou encore la Limited Partnership d'Angleterre, inspirée de la législation continentale sur les sociétés en commandite et reconnue par la Limited Partnership Act de 1907.

Quelle que soit la structure juridique de ces phénomènes commerciaux, elle provient toujours de sources faciles à repérer; une classification des différentes structures peut simplifier grandement, par la suite, les efforts de clarification des ambiguités juridiques et linguistiques de l'heure, conséquentes à la prolifération d'entreprises de toutes sortes.

Le travail que nous nous proposons doit donc se fonder sur un ensemble de notions juridiques puisées aux sources contemporaine et historique, toutes deux essentielles pour comprendre le vocabulaire actuel en droit commercial. Lorsque nous aurons décrit la situation juridique des institutions et leurs techniques d'organisation, il deviendra d'autant plus facile de faire œuvre descriptive en linguistique et, par la suite, nous passerons, là où il est possible de le faire, à la standardisation des termes du lexique juridique du commerce.

Nous étudierons d'abord le concept de la personnalité morale, ensuite, sous leur angle historique, trois types d'entreprises : la compagnie, la corporation d'inspiration anglaise et la sociéte; enfin, nous verrons les retombées de ces données sur la pratique commerciale au Québec. 
On remarquera, sans doute, que le présent exposé ne s'intéresse qu'aux sociétés commerciales et laisse de côté les sociétés civiles; ce n'est pas à cause d'un manque d'intérêt, mais plutôt parce que la nature non commerciale de ces sociétés les exclut d'office de notre thème : les entreprises commerciales.

\section{LA PERSONNE MORALE}

Pour autant que les concepts de personnalité morale en droit civil et de body corporate and politic ${ }^{1}$ en droit anglais soient analogues, ils recouvrent de fait deux réalités dont on constate les différences quand il s'agit de les mettre en application. Dans les deux cas, tant que l'on n'a pas à tenir compte de situations particulières, on peut définir la personne morale ou le body corporate and politic ainsi : "Groupement de personnes ou de biens ayant la personnalité juridique, et étant par conséquent titulaire de droits et obligations ${ }^{2}$.

En droit civil, le régime juridique des personnes morales s'étend aussi bien au droit public qu'au droit privé. À titre d'exemple, en droit public, on trouve diverses catégories, soit l'État, les départements, les communes et les établissements ; par ailleurs, en droit privé, on compte les associations, les congrégations, les fondations, les ordres et les sociétés. Dans chacune des catégories, la capacité de la personne morale est différente et c'est la loi qui précise son régime. De manière générale, on reconnaît à la personne morale les caractéristiques suivantes :

- elle naît, vit et disparaît, d'où la nécessité, lors de la disparition, de procéder à la dévolution des biens ;

- elle a le droit d'agir en justice;

- elle est susceptible d'engager sa responsabilité civile et, dans certains cas, sa responsabilité pénale ; nalité ${ }^{3}$

- elle a, en plus d'une existence juridique, un nom, un domicile, une natio-

En Common law, outre ces mêmes qualités, on trouve souvent une précision supplémentaire concernant la responsabilité limitée des membres d'un body corporate and politic, ce qui facilite le glissement du concept de la corporation, technique d'organisation, vers celui de la corporation, structure commerciale. Nous verrons plus loin que cette confusion de la terminologie anglaise, entre la corporation en tant que personne morale et la corporation en tant que société commerciale, constitue une source intarissable de quiproquos.

Si le texte législatif sert comme point de départ autant pour la personne morale que pour le body corporate and politic, de part et d'autre, droit civil et Common law ne s'expriment pas de la même manière pour les décrire. En effet,

1. On trouve, en droit anglais, trois tèrmes pour désigner cette réalité : corporation, corporate body et body corporate and politic. Pour faciliter le présent exposé, lequel est d'ordre juridique, nous préférons l'emploi de body corporate and politic, ce qui nous permet d'utiliser corporation pour decrire l'organisation du commerce.

2. Raymond Guillien et Jean Vincent, Lexique de termes juridiques, Paris, Dalloz, 1972,

p. 252

3. Michel de Juglart et al., Leçons de droit civil, les personnes, Paris, Editions Montchrestien, 1972, p. 620 et 621. 
mallgré l'existence indéniable du body corporate and politic, il n'existe pas en droit anglais de «codification» de ce concept; telle qu'on en trouve en régime civiliste, comme dans le Code civil du Québec :

Toute corporation légalement constituée forme une personne fictive ${ }^{4}$ ou morale dont l'existence et la successibilité sont perpétuelles, ou quelquefois pour un temps défini seulement, et qui est capable de certains droits et sujette à certaines obligations ${ }^{5}$.

ou encore, dans le Code civil de la Louisiane :

Une corporation est un corps intellectuel, créé par la loi, composé de plusieurs individus réunis sous un nom commun, dont les membres se succèdent de manière que le corps demeure toujours le même malgré le changement des individus, et qui, pour certains objets, est considéré comme une personne naturelle ${ }^{\mathrm{B}}$.

Il vaut la peine de mettre en relief l'originalité de ces deux citations en ce sens que les codificateurs, tant au Québec qu'en Louisiane, ont décidé d'intégrer le concept de la « corporation $\gg$ dans leur code civil, là où le code civil français omet d'en parler. Il semblerait que la raison de ce choix soit fondée sur le refus des civilistes français de considérer des propos sur la « corporation 》 comme pertinents en droit civil '. C'est cette décision qui est à l'origine d'une bifurcation fondamentale entre la méthode civiliste du Québec et de la Louisiane, et celle de l'Europe. Cela explique également nombre d'innovations terminologiques que certains qualifient de barbarismes dans les usages québécois; l'assise institutionnelle du droit québécois est un hybride et les langues qui le décrivent reflètent cet état. Cette question fait l'objet d'une étude plus approfondie sous la rubrique de * la personne morale en droit québécois ».

Dans le Common law, à défaut de principes codifiés, chaque texte législatif vient, par l'éclairage nouveau sous lequel il l'aborde, enrichir le concept de body corporate and politic. C'est ainsi que dans la Loi sur. les sociétés commerciales canadiennes, on précise dans le texte anglais que :

A corporation has the capacity and, subject to this Act, the rights, powers and privileges of a natural person ${ }^{8}$.

4. Bien que l'on dise que la personnalité morale est une fiction juridique, notion contestée de nos jours, on ne voit presque jamais dans la documentation contemporaine le terme \& personne fictive 》 pour désigner une personne morale. L'origine de \& personne fictive 》 semblerait remonter soit aux écrits du juriste Bartole, soit à une expression latine de droit ecclésiastique persona ficta, ce qui peut servir d'indice des sources qui ont inspiré les codificateurs québécois entre 1859 et 1864

5. Code civil du Québec, art. 352.

6. Code civil de la Louisiane de 1825, art. 418. Cet articlè est identique à l'article 427 de l'actuel Code civil de la Louisiane (Louisiana Civil Code, éd. Joseph Dainow, St. Paul, Minn., West Publishing Co., 1972; Compiled Edition).

7. John P. Richert et E. Suzanne Richert, The Impact of the Civil Code of Louisiana upon the Civil Code of Québec 1866 , in Themis (1973), 8 R.J.T., no 3, p. 512.

8. S.C.R. 1975 , c. 33, art. 15. - On remarquera que la version française de cet article vient souligner l'échec auquel est youée toute tentative d'une francișation superficielle des textes législatifs; la voici :

La société a, sous réserve de là présente loi, la capacité d'une personne physique. Manifestement, le désir de produire une image française l'a emporté sur la nécessité d'implanter un vocabulaire français qui respecterait les nuances de cette langue. Car l'assimilation de la capacité d'une société, donc par définition une personne morale, à une personne physique, constitue une.entorse originale aux pratiques juridiques habituelles... 
Dans ce cas précis, on sent le souci du législateur d'accorder à cette corporation, à ce body corporate and politic, des pouvoirs extrêmement vastes; plus loin dans ce texte, le législateur greffe cependant au principe si généreux tout un assortiment de restrictions pour réduire le rayon d'action d'une telle «personne physique s.

A peu de choses près, on remarque l'utilisation d'une formule semblable dans la Loi instituant la corporation commerciale canadienne. À l'article 3 , on crée la corporation et de là jusqu'à l'article 12 , on définit ses pouvoirs et ses devoirs, procédé tout à fait normal dans une loi établissant un organe administratif.

Suivant la conception traditionnelle régissant la création d'un body corporate and politic, il est à remarquer que seule la puissance politique est habilitée à déterminer si un groupement peut ou non bénéficier de ce statut particulier. Sur ce point, le droit civil semble parfois un tant soit peu moins formaliste, car il admet dans certaines circonstances l'existence de « personnes morales $\gg$ qui ne doivent pas leur création à un acte législatif ?.

Dès que le texte de loi entre en vigueur, son interprétation relève des tribunaux, à moins que le législateur n'y revienne, à une date ultérieure, pour faire des retouches par voie de modifications, ce qui arrive à chaque session de l'Assemblée. L'interprétation judiciaire exerce en Common law une influence considérable sur la vie d'une loi. Pour s'en rendre compte, il suffit de rappeler une cause importante de la jurisprudence québécoise ${ }^{10}$, celle de la Society Brand Clothes Ltd. v. Amalgamated Clothing Workers of America, (1931) R.C.S. 321. En l'occurrence, un groupement de travailleurs désireux d'ester en justice se vit refuser l'accès au tribunal en tant qu' \& association dotée de la personnalité morale », et ceci en raison du fait que les travailleurs ne s'étaient pas réunis au sein d'une association reconnue par la loi. Cette décision a marqué un tournant dans le droit québécois, car le législateur a par la suite ajouté au Code de procédure civile l'article 60 , lequel précise :

Tout groupement de personnes formé pour la poursuite d'un but commun dans la province, mais qui n'y jouit pas de la personnalité civile ni ne constitue une société au sens du Code civil, peut néanmoins ester en justice pour se défendre aux actions portées contre lui.

Tel groupement peut aussi se porter demandeur, s'il dépose au greffe du tribunal, avec l'acte introductif d'instance, un certificat du Commissaire enquêteur en chef en vertu du Code du travail, attestant qu'il constitue une association de salariés au sens du Code du travail.

Il s'agit donc toujours d'un groupement et non d'une personne morale, ce qui souligne une fois de plus l'importance qu'un droit inspiré du Common law attache

9. Ce fut le cas notamment des sociétés civiles dont le code civil français ne précise pas le statut. Or, c'est grâce à une construction jurisprudentielle que l'on reconnaît aux sociétés civiles là personnalité morale en vertu des articles 1845 et suivants du Code civil. Juglart, op. cit., p. 632.

10. En législation sur le travail tout comme pour les personnes morales, le droit positif du Québec est à tel point d'inspiration anglo-américaine que nous pouvons le citer en tant

...... qu'exemple du fonctionnement du Common law...Cette démarche. n'est valable que pour certains cas isolés, dont celui-ci. 
à la reconnaissance, par la puissance politique, du body corporate and politic, au moyen d'un acte législatif.

Il ne suffit pas, pour bien comparer la personne morale et le body corporate, d'agencer volume pour volume le contenu de l'une et de l'autre; il faut de plus sentir l'attitude de ces droits respectifs à l'égard de ces «êtres». Que ce soit en France ou en Angleterre, le commentaire suivant résume bien un courant de pensée européen :

L'histoire des personnes morales est celle de la lutte menée contre elles par l'Etat. Qu'il s'agisse de groupements ou d'œuvres, par la puissance politique, économique, financière qu'elles représentent, les personnes morales apparaissent redoutables à l'Etat qui, consciemment ou inconsciemment, souhaite n'avoir en face de lui que des individus faibles, donc isolés. Mais, de plus en plus, l'homme, sur quelque plan que ce soit, est condamné à l'inaction s'il est seul, s'il ne peut franchir les bornes de sa brève existence, s'il ne peut se dépasser dans le temps et dans l'espace. Or, l'Etat a besoin d'une activité féconde des citoyens; il ne saurait vivre s'il les stérilise en combattant les personnes morales. Mais, s'il ne lutte pas contre elles, il risque de perdre par elles toute autorité. Un équilibre doit donc être cherché. Dans quelle mesure est-il aujourd'hui réalisé en France ${ }^{11}$ ?

C'est dans les colonies d'Amérique que les personnes morales semblent ne plus porter le stigmate d'être un point de repli pour ceux qui sont hostiles à l'autorité de la puissance politique. Car ce que l'Europe a cherché à étouffer dans l'auf, l'Amérique y laisse libre cours ; cette floraison de personnes morales culmine à la fin du xix siècle dans un âge d'or de colossales sociétés que l'on dénomme des trusts, vastes agglomérations de capitaux et de biens constituées pour dominer d'importants secteurs de l'économie.

Dans le sillage tracé par ces entreprises démesurées, on voit la naissance et le développement de deux autres sortes de personnes morales, qui s'accaparent alors leur part de l'économie : les syndicats de travailleurs et les innombrables agences gouvernementales qui réglementent à la fois les sociétés et les syndicats.

C'est ainsi que la personne morale semble atteindre l'apogée de sa grandeur ou de sa décadence, devenant dans bien des cas une société parallèle, une institution commerciale, gouvernementale ou ouvrière où les membres s'occupent d'abord des intérêts de leur organisme.

Il reste enfin une dernière difficulté qui vient perturber la compréhension, que ce soit entre traducteurs, juristes ou simples profanes, c'est le dédale de la terminologie, surtout celle du Common law. En comparaison, le vocabulaire du droit civil est relativement facile, car on dit que tout être jouit de la personnalité juridique et que l'on désigne parmi ces êtres des personnes physiques et des personnes morales, selon certaines caractéristiques propres aux unes et aux autres. En Common law, la même réalité s'exprime par un choix d'expressions, telles : \& persons, groups and corporations \$, \& natural persons and bodies corporate and politic », individuals and legal, artificial or ideal persons », " physical persons and corporate or incorporated bodies $>. .$. et ces expressions ne sont nullement

11 i: .Juglart, op. cit . p: 613. 
figées, car selon le régime juridique où l'on se trouve, on rencontre toutes les combinaisons et permutations possibles.

\section{LA SOCIETE COMMERCIALE}

\section{Aperçu historique}

Le droit romain définissait la société comme une entente entre deux ou plusieurs personnes en vue de réaliser un objectif commun. On qualifiait d'affectio societatis des associés la volonté de séparer de leurs intérêts propres leur but commun par l'établissement d'un contrat synallagmatique formé intuitu personae, c'est-à-dire en considération de la personnalité du cocontractant.

Sous sa forme primitive, la société regroupait tout l'actif des associés (societas omnium bonorum), ce qui ne dure qu'un certain temps dans l'Antiquité, car le terme «société \& en est venu à comprendre toute entente visant une activité en commun, grande ou petite et de courte ou longue durée. Dans cette société de jadis, chaque associé devait fournir un apport en capital ou en industrie et, en principe, les devoirs des associés étaient identiques, ainsi, on ne reconnaissait qu'une action, l'actio pro socio, c'est-à-dire la capacité d'un associé d'obliger les autres associés à exécuter leurs termes du contrat de la sociêté.

Or, à l'encontre de la pratique contemporaine, ce contrat de société ne liait que les associés entre eux. Il n'ouvrait pas à un tiers le droit de poursuivre un associé autre que celui avec lequel il avait contracté ; autrement dit, en droit romain, une obligation était strictement de nature personnelle ${ }^{12}$. Par contre, de nos jours, les lois qui traitent des sociétés précisent que les associés (dans une société en nom collectif) sont responsables solidairement et indéfiniment sur leur propre patrimoine des dettes de la sociéte : That partners are jointly and severally liable for debts of the partnership and assets individually owned are subject to execution to satisfy any such debts. Mais voilà des propos qui devancent notre récit.

Ce n'est qu'à la Renaissance, et plus précisément à Venise, au XII ${ }^{\mathrm{e}}$ siècle, que l'on constate une véritable évolution dans la technique de constitution des sociétés. Il s'agit d'une innovation de la part de commerçants, soucieux comme toujours de réunir les capitaux indispensables à la poursuite de leurs affaires. Elle s'appelle la société en commandite et elle se distingue de son prédécesseur du droit romain par la coexistence de deux catégories d'associés : les commandités, responsables indéfiniment des dettes de la société, et les commanditaires, responsables jusqu'à concurrence de leur apport. Ces derniers deviennent de véritables associés, partageant les risques et les bénéfices, et non de simples bailleurs de fonds. Cette astuce permet à bon nombre de marchands de Venise de solliciter des finances auprès de sources habituellement inaccessibles, comme les nobles et le clergé. Malgré ce changement important, la société telle que la connaissait le droit romain subsiste encore, à la différence notable que chaque associé engage tous les autres vis-à-vis des tiers, et peut disposer de l'actif commun.

12. Barry Nicolas, An Introduction to Roman Law, Oxford, Clareadon Press, 1962, p. 185. 
Dès cette époque lointaine, on remarque la division des sociétés en deux catégories qui donneront plus tard naissance aux deux types dominants de sociétés commerciales dans l'Occident d'aujourd'hui : les sociétés de personnes (société de type romain) et les sociétés de capitaux (société en commandite... par actions). Pendant et après la Renaissance, la terminologie utilisée pour décrire ces entités a connu des variations. C'est ainsi qu'en France, dans l'Ordonnance sur le commerce de 1673 , on consacrait diverses dispositions aux \& sociétés générales * (en nom collectif), aux commandites (simples) et aux sociétés anonymes (en participation). Toutefois, cette ordonnance ne faisait aucun état de la société par actions, bien qu'on témoignât à cette époque de l'apparition des compagnies royales à caractère semi-public. C'est donc dire que naguère les droits français et anglais se ressemblaient à bien des égards et que l'écart qui devait les séparer n'intervint que plus tard, au moment de la Révolution française.

Nous avons vu qu'au cours des siècles, il y a eu flottement dans les appellations utilisées pour décrire les différentes sociétés. A partir de quel moment voit-on une fixation de ces usages? La terminologie actuelle date surtout du Code de commerce de 1807, où l'on découvre cinq types de sociétés :

1. La société en nom collectif (art. 20 à 22);

2. La société en commandite simple (art. 23 à 28);

3. La société anonyme (art. 29 à 37 );

4. La société en commandite par actions (art. 38);

5. La société en participation (art. 47 à 50);

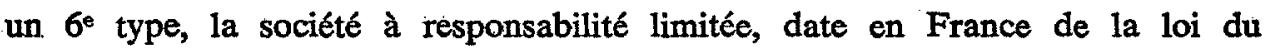
7 mars $1925^{18}$.

Quant à l'expression société par actions, le droit français s'en servait à l'occasion comme terme générique, comparable à société de capitaux. Ce n'est que par la loi du 24 juillet 1966 que l'expression société par actions accède au rang d'une « catégorie juridique ».

Un autre terme, aujourd'hui devenu archaĩque, qui mérite une attention passagère, c'est la société par intérêts, synonyme de société de personnes. Par ailleurs, au xvI ${ }^{\mathrm{e}}$ siècle, dans son Parfait Négociant, Savary parle des sociétés * collectives > (en nom collectif), en commandite (simples) et anonymes (en participation) ${ }^{14}$.

Dans la liste ci-dessus, on voit apparaître dans le Code de commerce de 1807, deux changements majeurs, c'est-à-dire l'introduction de la société en participation et la reconnaissance de la société en commandite par actions.

Il y a désormais deux formes de sociétés par actions : la scommandite par actions \& qui est libre, la \& société anonyme \& qui doit être autorisée. Cette expression, société anonyme, est empruntée à l'ancienne langue juridique, mais elle change de sens. Elle désignait dans l'ancien droit la

13. René Rodière et Roger Houin, Précis de droit commercial, Paris, Dalloz, 1970, p. 332. 14. Ibid., p. 332 . 
participation occulte. Elle va maintenant désigner la société dans laquelle il n'y a pas d'associé responsable, donc pas de raison sociale ${ }^{15}$.

Or, bien que le Code de commerce créât la société anonyme, celle-ci ne devait connaître qu'un succès fort médiocre auprès des hommes d'affaires, vu l'exigence d'une autorisation préalable dont le gouvernement l'avait encombrée. Les hommes d'affaires préféraient, par contre, exploiter les ressources de la société en commandite par actions, ce qui leur permettait, sur simple inscription auprès d'un tribunal de commerce, de constituer une société. En outre, dès 1832; la Cour de Cassation reconnaît à ces sociétés le droit de faire coter leurs actions dans les Bourses de valeurs, tout comme les sociétés anonymes : c'est l'époque de la «fièvre des commandités».

Lors de la seconde grande poussée du capitalisme sous le Second Empire, on verra reparaître (une) floraison dé commandites. Il s'en crée quatre ou cinq cents chaque année et seulement une douzaine de sociétés anonymes. La liberté qui leur est accordée permet aux fondateurs de rafler l'épargne. On voit circuler des actions de cinquante et de vingt francs; on en vit même de cinq francs! Il fallut réglementer la commandite par actions par la grande loi du 17 juillet 1856 et; dix ans après, à nouveau par la loi du 24 juillet 1867.

Cependant la proclamation obstinée de la liberté pour les sociétés anonymes ne cessait pas. Il y avait maintenant une raison nouvelle. L'Angleterre dictait notre politique économique. Or les compagnies anglaises, soumises autrefois à l'agrément du Parlement ou de la Couronne, venaient d'obtenir en 1856 leur incorporation par un simple enregistrement. Qui plus est, elles pouvaient poursuivre leur exploitation en France, car la loi du 30 mai 1857 , votée pour les sociétés belges, permettait d'accorder, par décret impérial, la même faveur à tous autres pays. Le traité du 30 avril 1862 l'avait accordée à l'Angleterre. Les compagnies anglaises librement formées seraient-elles donc libres chez nous, alors que les sociétés anonymes françaises ne pouvaient être créées sans autorisation ${ }^{16}$ ?

C'est alors, en imitant la Joint-Stock Companies Act de 1856, que les législateurs français donnent naissance à une créature étrange baptisée " société à responsabilité limitée», ou plus familièrement "société anonyme au petit pied ${ }^{17}$ ", entité qui n'avait rien à voir avec la société du même nom, issue de la loi du 7 mars 1925. Elle ne vivra que quatre ans. Voilà donc que 55 ans après la promulgation du Code de commerce, la France vacille sur la question de se doter d'une structure juridique répondant aux besoins commerciaux des entrepreneurs français ; il faudra encore quatre ans et une autre poussée des Anglais pour que cela se réalise.

La loi du 24 juillet 1867 [...] a été poussée à la suppression de l'autorisation préalable par cette raison « que, en vertu de la législation et des traités, les sociétés anglaises à responsabilité limitée, véritables sociétés anonymes libres, peuvent s'établir en France sans être astreintes à obtenir l'autorisation de l'État. Comment l'imposer à nos nationaux ${ }^{18}$ ?

Une fois votée, cette loi du 24 juillet 1867 demeure la norme en droit social jusqu'au 24 juillet 1966, jour où l'Assemblée nationale la remplace par 15. PauI Didier, Droit commercial, les entreprises, coll. Thémis, Paris; P.U.F., 1972, p. 69. 16. Ibid., p. 70 .

17. Ibid., p. 71 .

18. Paul Didier, Droit commercial, coll. Thémis, Paris, P.U.F., 1970, p. 436, . 
une nouvelle loi sur les sociétés commerciales, événement qui constitue une mise à jour importante pour les sociétés françaises.

Il est une distinction, utilisée jadis comme aujourd'hui pour classer une société dans l'une ou l'autre des catégories de société de personnes ou de société de capitaux : c'est la négociabilité des titres. Dans les sociétés de personnes, c'est-à-dire en nom collectif, en commandite simple, à responsabilité limitée ou en participation, on reconnaît un trait commun, le caractère incessible des parts. Quant aux sociétés de capitaux, c'est-à-dire anonymes ou en commandite par actions, elles n'attachent pas d'intérêt à la personne et les actions sont librement cessibles.

Toutefois, le critère de la négociabilité des titres n'est guère étanche, car il y a des titres cessibles qui circulent dans les sociétés de personnes et des titres incessibles qui meublent les archives des associés réunis en société de capitaux. Quioi qu'il en soit, le principe juridique demeure formel : tout le reste dépend des intentions des commerçants qui constituent une société.

Une observation sur la nature contractuelle des sociétés mérite notre attention, surtout en raison des rapprochements que fait l'auteur, Paul Didier, entre le droit et la linguistique. Le juriste se demande parfois : la société est-elle un contrat ou une institution ?

Les partisans de la thèse contractuelle s'appuient sur la lettre même du Code civil ( \& la société est le contrat »), les tenants de la thèse institutionnelle font état de l'évolution postérieure du Code. La controverse reste largement académique.

Probablement, les juristes gagneraient à utiliser ici une distinction $\mathrm{du}$ genre de celle que les linguistes font entre ce qu'ils appellent la Langue et la Parole. Admise dans les disciplines juridiques, cette distinction suggérerait une nouvelle distribution du couple institution/contrat. Au lieu d'être opposés, ces termes désigneraient les deux faces de tout phénomène juridique : la face institutionnelle, sociale (la Langue des linguistes) et la face contractuelle, individuelle (la Parole). Il y aurait alors lieu d'examiner, dans le discours juridique, la part de la langue (institution) et de la parole (contrat), la répartition n'étant pas la même pour toutes les institutions (ni toutes les langues quil laissent, selon le cas, une grande liberté au sujet parlant ou au contraire le contraignent fortement dans la construction des phrases ou des mots) ${ }^{19}$.

Voilà un ensemble de considérations qui constitue un apport précieux aux réflexions sur ce qu'est la \& signification juridique \& d'un terme.

\section{Caractéristiques}

Pour mieux comprendre ce qui précède, nous dégageons ci-dessous les grandes lignes juridiques des principales sociétés commerciales reconnues en droit français et en droit belge ${ }^{20}$ :

19. Paul Didier, Droit commercial, p. 300.

20. : Sir M.S. Amos et F.P. Walton, Introduction to French Law, $2^{\circ}$ ed., Oxford, Clarendon, 1963 , p. 345-355.

Loi 66-537 du 24 juillet 1966 sur les sociétés commerciales s in Code de Commerce, a.:- Paris, Dalloz, 1977-78. 
1. La société en nom collectif

1.1 Constitution : minimum de deux personnes;

1.2 Nom : raison sociale, suivie de « et Cie»;

1.3 Titres : parts incessibles;

1.4 Responsabilité : indéfinie et solidaire des associés;

1.5 Gestion : associés.

2. La société en commandite simple

2.1 Constitution : un ou plusieurs commandités et un ou plusieurs commanditaires ;

2.2 Nom : raison sociale, suivie de et Cie \$;

2.3 Titres : parts incessibles;

2.4 Responsabilité : indéfinie et solidaire des commandités, limitée jusqu'à concurrence de l'apport des commanditaires ;

2.5 Gestion : commandités.

3. La société à responsabilité limitée

3.1 Constitution : minimum de deux personnes;

3.2 Nom : dénomination sociale, suivie des mots : «ociété à responsabilité limitée » ou des initiales S.A.R.L.;

3.3 Titres : parts incessibles, sauf exception;

3.4 Responsabilité : limitée jusqu’à concurrence de l'apport des associés ;

3.5 Gestion : associés.

N.B. En Belgique, on dit une société de personnes à responsabilité limitée.

Il est intéressant de noter que lorsqu'on met en regard les régimes juridiques distincts qui, avant la réforme de 1966, régissaient la société anonyme "fermée * et la société à responsabilité limitée, on se trouve en présence de deux entités qui se ressemblent passablement quant à leur fonctionnement. Ce sujet mériterait une étude plus poussée pour dégager les répercussions juridiques et linguistiques qu'un tel partage peut provoquer.

\section{Les sociétés par actions}

Selon la loi 66-537 du 24 juillet 1966, il existe deux catégories de sociétés par actions : la société anonyme et la société en commandite par actions.

\subsection{La société anonyme}

4.1.1 Constitution : minimum de sept personnes;

4.1.2 Nom : dénomination sociale suivie du sigle S.A.;

4.1.3 Titres : actions cessibles;

4.1.4 Responsabilité : limitée jusqu'à concurrence de l'apport de chaque actionnaire ;

4.1.5 Gestion : directoire, conseil de surveillance, assemblée générale. 
N.B. On distingue également entre les sociétés fermées et les sociétés ouvertes. Les sociétés fermées ne peuvent pas faire appel public à l'épargne et elles ont un nombre limité d'actionnaires; leur capital social minimum est de l'ordre de 100000 francs. Par contre, elles subissent moins de contrôles gouvernementaux que les sociétés ouvertes. Celles-ci font appel public à l'épargne et sont contraintes à une réglementation plus sévère en matière de publicité et de recours à des institutions financières; leur capital social minimum est de l'ordre de 500000 francs. Généralement, les actions d'une société ouverte sont inscrites à la cote officielle d'une Bourse de valeurs.

\subsection{La société en commandite par actions}

4.2.1 Constitution : un ou plusieurs commandités et un ou plusieurs commanditaires ;

4.2.2 Nom : dénomination sociale ;

4.2.3 Titres : actions cessibles;

4.2.4 Responsabilité : indéfinie et solidaire de la part des commandités - limitée jusqu'à concurrence de l'apport des commanditaires et des actionnaires;

4.2.5 Gestion : commandités.

Selon l'article 5 de la loi de 1966 sur les sociétés commerciales : \& Les sociétés commerciales jouissent de la personnalité morale à dater de leur immatriculation au registre de commerce. Il n'y a que la société ou l'association en participation, anciennement désignée comme société anonyme, qui ne jouisse pas de cette qualité.

Un autre point capital qu'il convient de signaler, c'est que le droit civil ignore le concept anglais de "sceau de la compagnie (ou société) », ce qui explique en partie l'importance que ce droit attache à la raison ou à la dénomination sociale pour établir l'identité de la société ${ }^{21}$. De nos jours, on constate d'ailleurs qu'en droit commercial anglais, l'usage du sceau de la compagnie a souffert tant d'exceptions que la valeur de cette pratique semble devenue tout à fait hypothétique ${ }^{22}$.

\section{De la raison sociale d̀ la dénomination sociale}

L'origine de l'expression « raison sociale \$ doit être cherchée dans les livres de raison (de comptes) que tenaient les marchands des villes dès le Moyen Age, (étymologie : ratio, compte, d'où l'italien moderne : ragioniere, comptable). L'habitude s'est prise très tôt de n'inscrire que les noms de quelquesuns des associés et d'abréger la raison sociale par l'emploi de la formule \& Cie. Au siècle dernier, les auteurs ont voulu attacher une signification à cette abréviation, mais ils se sont divisés sur son interprétation ${ }^{23}$.

21. Amos et Walton, op. cit., p. 348.

22. The use of the company's name or seal is not a condition to the representation of companies in civil law, it merely indicates the representative's intention to contract on behalf of the company ". - d'après James Smith, - The Representation of Companies in Québec *, in Thémis (1973), 8 R.J.T., no 1, p. 29.

\& Article 23 - L'absence du sceau de la société sur tout document signé en son nom par l'un de ses administrateurs, dirigeants ou mandataires, ne le rend pas nul. $\gg-$ 23. Paul Didier; Droit commercial, p. 344. 
Comme nous l'avons vu plus haut, la raison sociale doit être l'attribut des sociétés de personnes et la dénomination sociale, celui des sociétés de capitaux, car tant qu'il y a des associés responsables, il faut les désigner, mais lorsque la société devient anonyme, il n'y a plus lieu de désigner des personnes. Voilà la théorie.

La pratique commerciale révèle de nombreux écarts à ce principe, trop longs à énumérer ici ${ }^{24}$. Il en résulte que le flottement dans l'usage des raisons sociales, des noms commerciaux et des dénominations sociales est devenu un phénomène qui échappe au contrôle des gouvernements.

En réalité, la raison sociale aspire, à travers l'étape du nom commercial, à devenir une dénomination sociale (ex. la raison sociale Rothschild \& Frères, devenue un nom commercial et aujourd'hui la dénomination sociale de la S.A. Banque Rothschild) ${ }^{25}$.

Au gouvernement du Québec, le Service des compagnies fait un effort digne de respect pour imposer un minimum de normalisation dans l'emploi des raisons ou dénominations sociales. Généralement ce service se conforme aux normes énoncées dans un document publié par l'Office de la langue française, intitulé : «Comment formuler une raison sociale en français $»$.

\section{Crise de la «société contemporaine»}

Bien qu'il semble contradictoire de l'affirmer, à une époque où le signifiant « société » est de plus en plus présent dans l'usage populaire, son signifié connaît une dilution généralisée qui rend l'emploi juridique du signifiant de plus en plus inefficace. Voici trois exemples :

1. Les nationalisations de nombreuses sociétés privées ont annoncé le début de l'ère des entreprises dites d'économie mixte. Toutefois, bien des entreprises nationalisées continuent de s'appeler «société anonyme», par exemple, la Société nationale des chemins de fer français.

Ses obligations de service public obnubilent son caractère juridique de société anonyme... On peut d'ailleurs en dire autant de toutes les sociétés dans lesquelles l'État est l'unique actionnaire et auxquelles on n'a conservé l'étiquette de société anonyme que par un hommage purement verbal à cette forme élective de capitalisme libéral ${ }^{26}$.

2. Lorsqu'on cherche la nature contractuelle de la société anonyme, il est toujours possible de remonter, par un raisonnement tortueux, à l'acte constitutif de la société pour insister sur la présence d'un contrat, même si, à la suite de sa constitution, cette caractéristique s'estompe derrière la nature institutionnelle et commerciale de la société. Il n'en va pas ainsi cependant de la société dite unipersonnelle. Bien qu'elle soit inconnue à l'heure actuelle dans la législation française, c'est une forme de société qui gagne la faveur de nombreux pays occidentaux et la France demeure parmi les derniers à la reconnaître. D'abord,

\footnotetext{
24. On trouve un développement intéressant à ce sujet dans Paul Didier, Droit commercial, p. 344, 5 et 6 .

25. llbid.,.p. 344

26. Rodière et Houin, op. cit., p. 605 .
} 
on voit bien que le raisonnement civiliste veut que cette forme de commerce soit un mode de personnalisation juridique d'un patrimoine affecté à une activité économique; mais ce n'est pas un contrat. Ensuite, bien des civilistes n'y ont vu qu'une échappatoire fiscale, indigne d'une considération plus poussée. Enfin, la popularité de cette société semble acquise, et sans doute un jour, l'Europe des Six (ou des Neuf?) imposera aux insoumis la \&société d'une personne... ou unipersonnelle? » Il n'est pas exclu que l'histoire se répète.

3. Au Canada, pour refouler l'emploi abusif du terme anglais corporation, on acclame l'utilisation de "quelque chose de plus français», en l'occurrence, société. Le motif sous-jacent à cette demande est certes valable, c'est-à-dire assurer l'épanouissement linguistique et culturel d'un groupe ethnique. Toutefois, certains efforts ressemblent davantage à un maquillage de mauvais aloi, et c'est notamment le cas du texte législatif créant la Société Radio-Canada, à l'article 34 où l'on voit :

Est instituée une corporation appelée Société Radio-Canada...

Ce genre de texte révèle le sort réservé aux signifiants et signifiés tels que "société »; ils deviennent une sorte de placebo lexical dont le rôle est plus d'ordre psychologique que juridique. Si tel est vrai pour les «grandes sociétés d'État »u Québec et du Canada, notre constat vaut également pour de nombreux particuliers qui constituent, en suivant les normes de Québec ou d'Ottawa, des sociétés dites à responsabilité limitée, ce qui revient à dire des «sociétés ayant statut juridique de corporation $»$.

En quittant le sujet des sociétés, il est utile de retenir ces quelques mots de Arnos et Walton :

... the society [...] is a generic term which has no counterpart in English legal terminology. It embraces both companies and partnerships, institutions which English law has tended to treat as two distinct and unrelated species, despite their similarity of function ${ }^{27}$.

\section{LA. COMPAGNIE}

\section{Aperçu historique}

Le terme «compagnie » est devenu, en français contemporain, un parent pauvre en terminologie juridique, son usage ne subsistant que dans la raison sociale des sociétés de personnes et dans les dénominations sociales de quelques vieilles et vénérables entreprises. Par contre, dans la législation d'Angleterre, le vocable company sert toujours à désigner ces grandes agglomérations commerciales qu'on appelle, en français international, des sociétés anonymes.

Pour saisir le pourquoi de l'état actuel des choses, tant en droit qu'en terminologie juridique, il faut revoir un peu l'histoire de ces entreprises.

En droit français, il est difficile de décrire les vicissitudes juridiques des compagnies royales de jadis, sans mettre en relief une ambiguïté qui caractérise

27. Amos et Walton, op. cit., p. 346. 
l'attitude des civilistes à l'égard de ces entités. Nous verrons par les citations suivantes que ces juristes n'ont d'abord pas tenu compte de la compagnie et l'ont ensuite assimilée de façon cavalière à la société de capitaux, ce qui n'est pas de nature à faciliter une description des réalités commerciales.

Dans notre ancienne France, on n'aurait pas eu l'idée que les hommes puissent faire le commerce sous le couvert de la société sans être euxmêmes des commerçants, ou tout au moins sans que l'un d'eux le soit. Il y avait bien des compagnies, mais c'était tout autre chose que des sociétés commerciales privées.

$\mathrm{La}$ royauté française eut au XVII' siècle le sentiment des services que pourraient rendre de grandes compagnies. Elle en permit la fondation, mais la création d'un être est un acte divin, à tout le moins royal. Une compagnie ne peut avoir de personnalité s'il n'y a pas, suivant l'expression anglaise, une incorporation. C'était en France une charte royale qui lui donnait la vie. Le roi ne la consentait que pour un grand intérêt public : l'exploitation des colonies, le commerce maritime et, plus tard, la banque et l'assurance. Mais si l'œuvre était grande, il lui donnait sa protection, s'y intéressait personnellement, accordait la noblesse à ceux qui y collaboraient, octroyait la naturalisation aux étrangers. Il faisait mieux et concédait à la compagnie des parcelles de son pouvoir souverain : les droits de guerre, de justice, de battre la monnaie.

Les anciens juristes ne s'occupaient guère de ces grandes compagnies. Savary se borne à en signaler l'existence ; Jousse et Pothier n'en parlent pas. Certains de nos contemporains leur ont reproché sans indulgence leur pauvreté d'esprit. Jugement trop sévère : les compagnies étaient hors du droit privé. Ceux qui commentaient l'Ordónnance sur le commerce n'avaient pas à s'occuper de ces corps qui, par la grâce royale, faisaient des opérations commerciales, mais ne figuraient pas dans l'ordre civil ${ }^{28}$.

Certes, c'est un point de vue qui se défend. Il n'en demeure pas moins cependant que lorsqu'on a senti la nécessité de rebaptiser le statut juridique de la compagnie, elle a pris place parmi les sociétés :

La première société anonyme officiellement reconnue comme telle est la Compagnie hollandaise des Indes orientales qui date de $1602{ }^{29}$.

Or, ce flottement juridique et terminologique qui existait depuis longtemps, ne se manifeste que pendant et après la Révolution française, alors qu'on cherche à instaurer, à l'aide du Code de commerce de 1807, un ordre nouveau en droit commercial français. Sous l'ancien régime, la compagnie, création du souverain, était née d'une constitution écrite qui \& prenait la forme d'une déclaration, d'un édit, de lettres patentes ou bien encore d'un arrêt du conseil, en bref, d'un acte à caractère législatif tirant son origine de la volonté royale ${ }^{30} \gg$.

$\mathrm{Vu}$ son origine royale, la compagnie avait un caractère public et recevait par charte royale la personnalité juridique ; de ce fait, les associés étaient dégagés de la responsabilité illimitée à l'égard des actes de la compagnie.

En France et en Angleterre, la formation et l'exploitation de ces firmes étaient, à tous égards, presque identiques, y compris les nombreux scandales qui

28. D'après Georges Ripert, cité par Paul Didier, Droit commercial, les entreprises, p. 67, 68.

29. D'après Sayous, cité par P. Didier, Droit commercial, p. 435.

30. Ibid., p. 312 . 
devaient ébranler les fondements de leurs économies et sonner le glas de cette forme de commerce. Grandioses et nombreuses étaient ces compagnies et parmi elles, on trouvait :

- la Compagnie du Canada,

- la Compagnie des Cent-Associés,

- la Compagnie des Indes orientales,

- la East India Company,

- la Compagnie de la Baie d'Hudson,

- la Royal African Company ${ }^{s 1}$,

ainsi que d'autres. En Angleterre, on les appelait joint-stock companies; leur capital provenait de sources privées et l'autorisation d'être one body corporate dérivait de la prérogative régalienne.

Pour une grande part, la colonisation de l'Amérique du Nord est, à l'origine, le produit de ces joint ventures ou participations, qui réunissaient les intérêts du souverain et ceux des commerçants.

Vers la fin du Xvir siècle, il existait un assez grand nombre de compagnies pour qu'on vît la naissance du commerce des valeurs mobilières, lequel engendra à son tour : spéculation effrénée, accaparement d'entreprises, rafle de l'épargne et, enfin, intervention des gouvernements. La première intervention draconienne eut lieu en 1720, à la suite de l'effondrement catastrophique de la South Seas Company, appelée familièrement le * South Seas Bubble». Pris de panique, le Parlement anglais adopta la \& Bubble Act», mesure législative qui prétendait interdire, à moins d'une autorisation préalable, la formation de compagnies à responsabilité limitée et le libre transfert des valeurs mobilières. Or, l'hiver ne dure qu'un temps et dès le printemps suivant, d'astucieux praticiens avaient trouvé le moyen de jumeler la société en nom collectif (partnership) et la fiducie (trust) pour créer la unincorporated joint-stock campany. C'était une forme hybride de société, fondée en vertu d'un contrat passé entre des associés, lesquels souscrivaient un capital social divisé en titres cessibles. Quoiqu'il fût illégal de limiter sa responsabilité, souvent le contrat stipulait que l'associé n'était tenu qu'à concurrence de tant d'argent.

Le contrat désignait également des fiduciaires (trustees), propriétaires réels des biens de la compagnie que les associés, devenus des beneficial owners, autorisaient à ester en justice au nom de la compagnie. A défaut d'être étanche, cet arrangement fournissait tout de même à ses auteurs une immunité quasi totale à l'endroit des créanciers, vu les énormes difficultés que les tiers auraient eues à saisir, par voie de procédure judiciaire, un corps d'associés en évolution continuelle.

Pour douteuse qu'elle fût, cette pratique ne suscita qu'une jurisprudence relativement faible et pendant les 105 années suivantes, l'Angleterre devait

31. Encyclopaedia Britannica : Macropaedia, ed. W. Benton et al., Toronto, Britannica, 1975, vol. 5 , p. 183.

Encyclopaedia Universalis, red. R. Aron et al., Paris, Universalis, 1973, vol. 15, p. 51. 
connaître un régime ambigu où, officiellement, on décourageait les joint-stock companies et, officieusement, on tolérait jusqu'à un certain point les unincorporated joint-stock companies.

En 1825, le Parlement abroge la « Bubble Act» et ne manifeste aucune intention de légiférer à nouveau en la matière; les litiges sont donc renvoyés aux tribunaux de droit commun. Cette situation durera dix-neuf ans; entre-temps, les pressions montent pour que l'on règle une fois pour toutes la question de la légitimité d'une compagnie par actions, reconnue par la puissance publique. Finalement, en 1844, les Anglais trouvent l'expression législative qui puisse le mieux convenir à la incorporated joint-stock company et cette année-là, le Párlement adopte la célèbre "Joint-Stock Companies Act », loi qui sert de base, par la suite, à toutes les compagnies commerciales à voir le jour. Selon cette loi, les fondateurs d'une compagnie peuvent désormais obtenir « l'incorporation » au moyen d'un simple «enregistrement», donc, un obstacle majeur vient de disparaître. La loi fournit également bien des précisions concernant le nombre d'associés nécessaires, la publicité et les états financiers. Douze ans plus tard, en 1856, on refond cette loi et le nouveau texte législatif, également intitulé « Joint-Stock Companies Act»; introduit quelques précisions :

- l'incorporation est le produit de l'enregistrement des statuts de la compagnie, déposé par un minimum de sept personnes,

- une compagnie doit afficher « Limited à la suite de son nom ${ }^{32}$.

Les grands principes établis, l'histoire de la législation sur les compagnies "devient, en Angleterre comme partout ailleurs, une suite sans fin de modifications ${ }^{33}$, de mises à jour et d'emprunts aux autres systèmes.

\section{Caractéristiques}

Il n'entre pas dans les usages du droit anglais de sérier sous une même rubrique des entités aussi distinctes que la «Limited Company » et la « Partnership ». Malgré ces usages vénérables, pour les fins de la présente étude, nous groupons et décrivons toutes les techniques d'organisation qui servent de charpente juridique aux entreprises commerciales.

Pour situer encore mieux la nature des entreprises anglaises, il est utile de rappeler les mots de Gower :

... the modern English business corporation has evolved from the unincorporated partnership, based on mutual agreement, rather than from the corporation, based on a grant from the State, and owes more to partnership principles than to rules posed on corporate personality. Thus we, in England, still do not talk about business corporation or about corporation law, but about companies and company law ${ }^{84}$.

32. Encyclopaedia Britannica, réd. W. Benton, Toronto, Britannica, 1971, p. 218-220.

33. Parmi les lois anglaises principales, on retient : - The Companies (consolidation) Act, 1908 :

- The Companies Act, 1929 ;

- The Companies Act, 1948 ;

- The Companies Act, 1967.

34. L.C.B. Gower, - Some Contrasts between British and American Corporation Law * in 19.69 Harvard Law Review, p. 1371-1372. 
Voici le détail de ces entreprises ${ }^{35}$ :

1. The (General) Partnership

1.1 Constitution : minimum 2 persons;

1.2 Nom : firm name;

1.3 Titres : non transferable shares;

1.4 Responsabilité : joint and several liability ;

1.5 Gestion : each partner is a general agent acting in the name of all partners.

N.B. En Angleterre, la «Companies Act 》 précise qu'à moins d'être constitué en compagnie, il est illégal pour un groupement de vingt personnes ou plus d'exploiter une affaire en vue de réaliser des bénéfices.

\section{The Limited Partnership}

2.1 Constitution : minimum of one general partner and one or more silent (sleeping or dormant) partners ;

2.2 Nom : firm name;

2.3 Titres : non transferable shares;

2.4 Responsabilite : unlimited for the general partner, limited to the amount contributed by the silent partner(s) ;

2.5 Gestion : general partner(s) only. If a silent partner actively manages the firm, he ceases to be «silent \$.

\section{The Limited Company or (Incorporated) Joint-Stock Company}

3.1 Constitution : depending upon the legal system, three sometimes seven or more persons ;

3.2 Nom : corporate name;

3.3 Titres : transferable shares ;

3.4 Responsabilité : liability limited to the amount paid for each share ;

3.5 Gestion : directors or board of directors;

$N . B$. En Angleterre, la loi de 1908 établit une distinction formelle entre les compagnies privées et les compagnies publiques. La constitution d'une compagnie privée ne pouvait être l'œuvre que de deux personnes; la compagnie publique devait en avoir sept. De plus, la compagnie privée fut contrainte à un maximum de 50 associés et à ne pas faire appel public à l'épargne. Par contre, cette compagnie bénéficiait de certaines exemptions en matière de publication de ses états financiers. D'après la loi de 1967, cette distinction entre compagnies privée et publique est abolie.

Aujourd'hui, on observe en dehors de ces structures traditionnelles une floraison de types nouveaux dans les compagnies anglaises et américaines ; il serait donc nécessaire de mettre au point une typologie nouvelle pour faire

35. P.G. Osborn, A Concise Law Dictionary, London, Sweet \& Maxwell, 1964, p. 58, 78, $195,234,235$. 
ressortir les traits communs ou distincts de ces entités. C'est ce que nous essayons de faire à l'endroit des corporations, sous la rubrique suivante.

\section{LA CORPORATION}

L'emploi du terme \& corporation pour désigner une société commerciale vient de l'usage américain de l'anglais. D'ailleurs, nous avons déjà vu que les Anglais, à travers toute leur histoire, ont préféré garder le mot compagnie même si du point de vue juridique, ils reconnaissent à cette entité le statut de body corporate. On peut donc dire que l'usage américain de corporation est un néologisme datant du XIX siècle et qu'il donne à ce signifiant deux signifiés :

1. Celui de body corporate and politic (personne morale),

2. Celui de commercial company (société commerciale).

Cette polysémie se révèle souvent fort encombrante lorsqu'on désire cerner la "vraie » nature juridique de la corporation. Il est également difficile de saisir à partir de quel moment l'usage américain a tacitement favorisé l'emploi de corporation aux dépens de compagnie, du fait que les auteurs contemporains ne parlent que des corporations, même quand il est question de la période coloniale. Or, lorsqu'on cite un nom de corporation de cette époque révolue, il s'agit toujours de company. Cette assimilation semble identique à celle que les Français font à l'égard de société et de compagnie.

\section{Aperçu historique}

La corporation, sous sa forme traditionnelle de personne morale, figurait dans la structure de nombreux organismes de l'Angleterre de la Renaissance. Dès le Xve siècle, le Common law précisait l'existence des corporations simples ou multiples (sole or aggregate). Une corporation simple désignait une entité dans laquelle une fonction est personnifiée, telle la corporation d'un évêque, d'un Grand vicaire ou encore d'un souverain. La corporation multiple désignait une entité dans laquelle on reconnaissait l'existence d'une personne autre que celle de ses membres, ce qui fournissait une structure administrative accessible aux municipalités et à certains corps de métiers.

Dès cette époque, l'on précise qu'en matière d'obligations et de transfert de propriété, la corporation ne se trouve liée qu'à la seule condition que l'empreinte de son sceau figure sur les contrats et les titres.

A l'aide de la structure corporative, des gens d'un même métier, soit apprentis, compagnons ou maitres (apprentices, journeymen or masters), pouvaient se constituer en association que l'on appelait un guild en Angleterre et aux Pays-Bas et, en France, une corporation. Il est fort intéressant à ce propos de constater qu'en France, le sens étymologique de corporation, c'est-à-dire - corporari : se grouper en corps \$, tomba rapidement de l'usage; seul subsista par la suite le sens qu'on trouve en français européen contemporain : "organisme social qui groupe tous les membres d'une même profession ${ }^{86}$. \$

36. LEXIS, dictionnaire de la langue française, Paris, Larousse, 1975, p. 412. 
A travers deux siècles d'évolution, le sort réservé à ce terme est étrange. Aujourd'hui, le francophone européen emploiera les mots "corporation 》, * corporatisme » ou «corporatiste \$ avec circonspection, car ils sont teintés d'une connotation politique de droite que certains contemporains qualifieraient de "poujadisme ». Tandis qu'à l'époque de la loi Le Chapelier ${ }^{37}$ votée le 14 juin 1791 pour supprimer les associations ouvrières, les corporations et surtout les compagnonnages symbolisaient davantage un mouvement prolétarien, donc de gauche selon certains : ce qui n'était autre chose que les balbutiements des syndicats de travailleurs que la bourgeoisie naissante avait d'ailleurs intérêt à circonscrire. Ainsi, le concept de la corporation passa de la gauche à la droite, tout en s'estompant dans la mémoire des Français : sa disparition ne fait plus aujourd'hui que figure de «triomphe * de la Révolution.

Ce glissement sémantique devient lourd de conséquences lorsqu'on considère les difficultés de communications, au sein de la francophonie, que provoque l'emploi canadien de * corporation $\gg$ dans le sens de société commerciale.

C'est au cours des $\mathrm{XIX}^{e}$ et $\mathrm{XX}^{\mathrm{e}}$ siècles que l'on constate, outre les formes corporatives déjà vues, un accroissement important dans la variété des corporations, notamment :

1. la corporation publique (ou politique), organisme créé par l'État pour réaliser certains objectifs, tel \$The United States Postal Service»;

2. Ia corporation privée, corps créé par un particulier pour son propre bénéfice. Ce genre de corps constitué peut s'adapter à un nombre illimité de fins.

À la fois technique d'organisation et structure administrative, la corporation est une réalité du droit anglo-américain qui met à rude épreuve toute description synthétique de ses applications. Tantôt commerciale, tantôt sociale, parfois ecclésiastique ou gouvernementale, la corporation a une qualité éthérée de passe-muraille qui fait qu'on la retrouve partout.

Aux États-Unis, il n'existe pas de loi fédérale sur les entreprises commerciales; ce domaine relève de l'initiative de chacun des cinquante États. Par contre, à l'endroit du commerce des valeurs mobilières, ce qui constitue une activité " inter-étatique », la législation fédérale est fort détaillée et son application est l'œuvre de la puissante \&. Securities and Exchange Commission», organisme quasi judiciaire créé en 1934 pour faire respecter la « Securities Exchange Act ${ }^{38}{ }$. Cette absence de loi fédérale se traduit par de nombreuses variations sur un même thème. En général, parmi les Etats, il existe un certain consensus pour déterminer ce qui constitue une entreprise, mais les modalités peuvent varier de zéro à l'infini.

À titre d'exemple, si l'on exige en Louisiane un capital social minimum, dans le Delaware, cette formalité semble inconnue; de plus, un intéressé peut

37. Désiré Brelingard et al., Histoire, le monde de 1715 d. 1870, Paris, Hachette, 1966, 38. 102-103.

38. Glen G. Munn, Encyclopaedia of Banking and Finance, ed. F.L. Garcia, Boston, Bankers Publishing Co., 1962, p. 671. 
demander, par correspondance, «l'incorporation» d'une société au Delaware, sans jamais y avoir mis les pieds! Derrière cette situation apparemment singulière réside le fait que le Delaware, petit Etat aux revenus modestes, tire grand profit d'une concentration sur son territoire de sièges sociaux auxquels il accorde de généreux avantages fiscaux.

\section{Caractéristiques}

Considérant la variété de structures possibles, donc l'impossibilité d'imposer une grille uniforme pour les classer, nous suivons la typologie de Hoghton ${ }^{39}$ selon laquelle il propose deux catégories aux contours flous.

\section{A. L'entreprise de capitaux d̀ caractère public}

Sous ce large chapeau, on peut abriter trois types d'entreprises qui possèdent toutes certains traits communs, notamment :

- une répartition bien définie entre la propriété et la maîtrise de l'entreprise ; à cet égard, les rôles respectifs des directeurs, des gérants et des actionnaires sont clairs.

- outre la traditionnelle recherche de profits, l'entreprise joue dans la communauté un rôle de «moteur social » dont il découle certaines responsabilités.

Selon Hoghton, ces trois types sont : les très grandes entreprises, les grandes entreprises et les entreprises de services.

1. Les très grandes entreprises. - On remarque dans cette catégorie un nombre restreint de vieilles entreprises dont les origines, pour certaines d'entre elles, remontent souvent à l'époque précédant la Guerre de Sécession. Elles sont à l'origine du développement de quelques grands secteurs : les chemins de fer, l'acier, puis plus tard, l'automobile et le pétrole. Du fait de leur envergure, l'administration en est devenue fort hiérarchisée ; leurs moyens financiers peuvent dépasser ceux de quelques États américains.

2. Les grandes entreprises. - Plus jeunes que les précédentes, ces entreprises se caractérisent par un engagement moindre vis-à-vis de la société et par une plus grande élasticité dans leur administration. Elles demeurent encore assez petites pour ne pas attirer les foudres des agences gouvernementales qui réglementent l'industrie.

3. Les entreprises de services. - Ce sont les dernières arrivées sur le marché américain; même si les services que fournissent ces entreprises ne sont pas nouveaux, leur façon de les présenter est originale. Elles ne datent souvent que de la Deuxième Guerre mondiale et, malgré cette jeunesse, elles ont réussi à dominer certains secteurs de l'économié.

39. Charles de Hoghton, The Company law, structure and reform in eleven countries, London, Allen \& Unwin Ltd., 1970, p. 54-57. 


\section{B. Les entreprises de capitaux à caractère privé}

À l'encontre des entreprises à caractère public, elles sont en général relativement jeunes. On n'y trouve pas de répartition de la propriété et de la maîtrise de l'entreprise parmi ses directeurs, ses gérants ou ses actionnaires. De même, il n'existe aucun engagement envers la collectivité, ce qui revient à dire que la réalisation de bénéfices occupe le premier plan des priorités de l'entreprise. Ces entreprises sont donc bien plus "agressives" dans le commerce.

Hoghton suggère trois catégories pour décrire ces entreprises : « enterprises », « ventures » et « wildcatters », et il leur reconnaît des critères moins étanches que ceux des entreprises à caractère public.

\section{LE QUÉBEC}

Le droit commercial du Québec s'inspire principalement de trois sources :

1. le droit français,

2. le droit anglais,

3. le droit américain.

$\mathrm{Si}$, dans un passé lointain, le droit français exerça une influence prépondérante sur les institutions juridiques et sociales du Québec, le droit anglais d'abord, puis le droit américain prirent la relève. Dans le domaine des sociétés commerciales, cependant, l'influence des droits français et anglais demeure la plus importante et s'explique dans une perspective historique.

En 1859, lorsqu'on nomma les trois commissaires ${ }^{40}$ chargés de l'élaboration et de la rédaction d'un code civil pour le Bas-Canada, ils durent faire œuvre de droit comparé du début de leurs travaux jusqu'à la fin, en novembre 1864. Dans le résultat de ces travaux, le Code civil de 1866 , deux questions retiennent l'attention aux fins de la présente étude :

1. Le traitement de la personne morale ou body corporate;

2. l'organisation du droit des sociétés.

\section{La personnalité morale}

Les codificateurs ont choisi de traiter des «bodies corporate » en suivant l'exemple du Code de la Louisiane, c'est-à-dire en les intégrant dans le Code civil, ce qui allait à l'encontre de la décision qu'avaient prise, en France, les rédacteurs du Code Napoléon. Voici leurs commentaires à ce sujet ${ }^{41}$ :

Ce titre (Des corporations) est tout nouveau; il ne se trouve pas au Code Napoléon, où l'on a entièrement omis le sujet des corporations; il n'en est

40. The committee [...] began its work in 1859, after the appointment of three members of the judiciary, Rene Edonard Caron, Auguste Norbert Caron and Charles Dewey Day, as Commissioners in charge of codification efforts. "- D'après John P. Richert et E. Suzanne Richert, The Impact of the Civil Code of Louisiana upon the Civil Code of Quebec of 1866\%, in Thémis (1973), 8 R.J.T., na 3, p. 504.

41. Rapport des codificateurs, Second rapport (1865), p. 228-229. 
parlé que d'une manière incidente et sous la désignation de communes et établissements publics, dans les articles suivants entr'autres, savoir : 910, à propos des donations et testaments, 2045 des transactions, et 2121 des hypothèques légales.

En recherchant la cause de cette omission, ce qu'on a trouvé de plus probable est ce qui se rencontre au $\mathrm{II}^{\mathrm{e}}$ volume de la concordance des codes par St-Joseph, p. 477 , (édit. in quarto), où parlant des articles 418 et 438 de la Louisiane, l'auteur dit : « toutes les dispositions (contenues dans ces articles) sont relatives aux corporations, à leur nature, à leur usage, à leurs privilèges, incapacités et dissolution; on n'a pas cru nécessaire de les reproduire, comme n'ayant aucun trait au droit civil proprement dit. » Ainsi, d'après cet auteur, les corporations ne feraient pas partie du droit civil proprement dit; elles dépendraient donc de cette partie du droit public nommé droit administratif.

Cette assertion a paru incorrecte. Il est vrai que les corporations doivent leur existence légale à l'autorité publique (royale ou législative). Une fois créées et organisées, elles deviennent personnes morales ou fictives, capables de certains droits et privilèges et tenues à certains devoirs et obligations ; mais ces droits et ces devoirs ne sont pas à tous égards ceux des personnes ordinaires; la nature même des corporations, leur objet et leur destination, sont les causes nécessaires de plusieurs de ces différences, tandis que d'autres procèdent des précautions que l'intérêt public a fait prendre contre les envahissements et les empiètements auxquels sont naturellement portés les corps en général, dont la responsabilité partagée entre les membres est moins fortement sentie par chacun d'eux.

Il fallait donc, par des règles particulières, pourvoir à ces objets exceptionnels, dont plusieurs ne peuvent tomber sous l'action directe du droit public. Ces règles tendant à organiser les rapports de ces êtres fictifs avec les autres membres de la société, ont dû être empruntées au droit civil, dont elles font partie tout aussi bien que celles qui régissent les droits, les obligations, les incapacités et les privilèges des mineurs, des absents, des insensés, etc.

L'omission au Code Napoléon de toutes les dispositions sur le sujet est due probablement au peu de cas que l'on faisait autrefois en France des corporations qui, avant et même depuis le code, étaient loin de jouir de l'importance qu'elles ont depuis longtemps acquise en Angleterre et dans quelques autres pays, où elles sont si variées et si nombreuses, et où les lois y relatives sont, par suite, en nombre proportionné.

Avant la révolution, en France, les pouvoirs législatifs et administratifs étant concentrés dans les mêmes mains, les «corporations 》 qui y existaient étaient créées et dirigées par le même pouvoir, ce qui simplifiait considérablement les règles sur le sujet; de là le petit nombre de ces règles que l'on trouve dans les livres avant cette époque.

Lorieux, dans son excellent traité sur la prérogative royale, (1 vol. p. 483), dit : \& En France, les principes, en ce qui concerne les corporations, sont en général fort mal compris et souvent méconnus, ou plutôt en cette matière il n'existe ni règles ni doctrines $» ;$ et il ajoute ensuite : « la législation nouvelle s'en est rarement occupée, et quant aux maximès anciennes, on les met complètement en oubli. $\gg$ Le chapitre 5 de cet ouvrage est ce qu'on trouve dans les livres français de plus intéressant sur les corporations, dont au reste, on paraît dernièrement s'être occupé davantage, si on en juge par le nombre de lois promulguées sur le sujet depuis 
l'adoption du Code. Pour remédier à la lacune qui y est ainsi laissée, les Commissaires ont préparé et soumettent le présent titre intitulé : «Des Corporations », à l'instar de celui qui se trouve au Code de la Louisiane, dont au reste, ils n'ont pu s'aider que médiocrement.

Cette décision des codificateurs devait servir de base à toute une structure jurisprudentielle; elle a mis à la disposition des futurs législateurs québécois un outil juridique aux multiples usages. Il est intéressant de voir, à pied d'œuvre, quelques échantillons législatifs où l'on a puisé dans ce « fonds commun » de la corporation. Toutes les lois suivantes autorisent une constitution en corporation.

- Loi des syndicats coopératifs, S.R.Q. 1964, c. 294, a. 3-12 ;

— Loi des sociétés coopératives agricoles, S.R.Q. 1964, c. 124, a. 4-8-9 ;

- Loi des associations coopératives, S.R.Q. 1964 , c. 292 , a. 8-14 ;

- Loi des caisses d'épargne et de crédit, S.R.Q. 1964, c. 293, a. 8-16 ;

- Loi des clubs de récréation, S.R.Q. 1964, c. 298, a. 3-7;

- Loi des clubs de chasse et de pêche, S.R.Q. 1964, c. 204, a. 1 ;

- Loi des fabriques, S.Q. 1965 , c. 76 et modifications.

En fait, il serait fastidieux de citer le nom de toutes les lois du Québec qui touchent de près ou de loin à la corporation. Il suffit, en effet, de citer brièvement feu le notaire Yves Caron qui remarqua un jour que plus d'un tiers des trois cents et quelques lois du Québec comportaient des disposition pertinentes aux corporations ${ }^{42}$.

Pour cerner avec précision les caractéristiques de la corporation selon le Code civil, $\mathrm{M}^{\mathrm{e}}$ André Couture propose l'explication suivante :

Ainsi, comme toute personne physique, la corporation doit porter un nom distinctif ${ }^{43}$. Elle possède un domicile, qu'on désigne sous le nom de siège social ${ }^{44}$. Elle est également titulaire d'un patrimoine ts et peut ester en justice en son nom ${ }^{46}$. Mais elle jouit de certains privilèges et souffre de diverses incapacités par rapport aux personnes physiques ${ }^{47}$.

Ces privilèges peuvent être soit spéciaux, soit naturels ${ }^{48}$. Les privilèges spéciaux sont ceux accordés spécialement à une corporation en particulier par sa charte ou par une loi particulière. Les privilèges naturels sont ceux qui lui résultent de sa nature de corporation, tel, par exemple, le privilège de la responsabilité limitée... ${ }^{49}$

Quant aux incapacités, elles sont également de deux ordres : légales ou naturelles ${ }^{50}$. Les incapacités légales sont celles qui peuvent être imposées par une loi particulière ou sa charte et celles imposées par la loi aux gens de mainmorte ${ }^{51}$. Notons que dans le cas des compagnies, cette dernière catégorie ne s'applique plus aujourd'hui : elles peuvent donc librement ac-

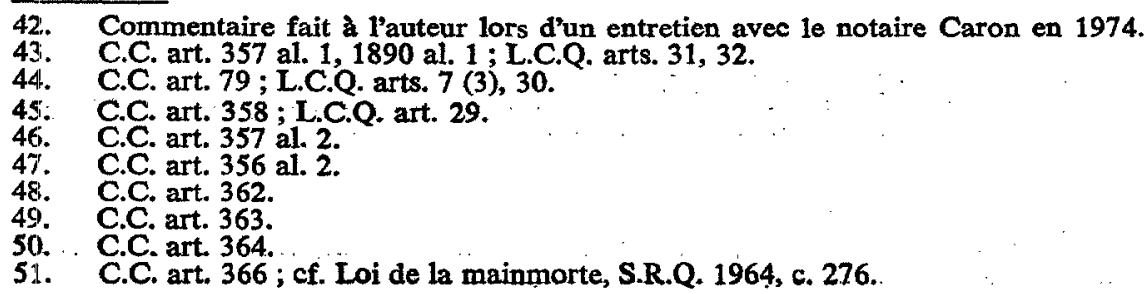


quérir et disposer des biens meubles et immeubles ${ }^{52}$. Quant aux incapacités naturelles, i.e. celles résultant de la nature même des corporations, le Code civil en donne une énumération à l'article 365 : les corporations ne peuvent être tuteurs, curateurs, exécuteurs testamentaires, témoins, jurés, gardiens, séquestres judiciaires. De même, elles ne peuvent être assignées personnellement, ni comparaître en justice autrement que par procureur ${ }^{53}$, ni poursuivre ou être poursuivies pour voies de fait.

On voit donc que si les corporations sont des personnes, elles sont des personnes particulières en ce sens que leur régime juridique s'inspire de celui des personnes mais est parfois modifié par des dispositions particulières ${ }^{54}$.

Dans son projet de Code civil ${ }^{55}$, l'Office de révision du code civil propose à l'endroit de l'ancien titre «Des Corporations » une notion inédite, celle de civiliser la corporation en droit québécois. Considérant ses contributions substantielles au droit positif, il ne pouvait être question de supprimer la corporation. Toutefois, la présence et surtout l'apparence de cette institution ont de quoi gêner un esprit civiliste et francophone, car les origines et le comportement juridique de la corporation recèlent encore un cachet de Common law ; bref, elle n'a pas été bien « naturalisée » en droit civil.

L'Office de révision du Code civil n'a pas voulu s'écarter d'une riche tradition, ni amputer la collectivité d'une institution originale qui lui rend de multiples services ; par ailleurs, il veut remettre en valeur la pensée civiliste, laquelle ne résistait pas toujours à la facilité d'emploi de la corporation. C'est ainsi que sous le titre des personnes morales, on en trouve une forme particulière : la corporation, qui obéit à des règles de droit civil. Ensuite, on passe aux personnes morales de droit public.

\section{Les entreprises commerciales}

$\mathrm{Au}$ Québec, un entrepreneur, désireux de donner à son commerce une structure juridique appropriée, peut choisir parmi une des trois formes suivantes :

1. La société en nom collectif ;

2. La société en commandite ;

3. La société par actions ${ }^{56}$.

Dans le Code civil, les deux premières catégories sont régies par les articles 1863 à 1888 ; par contre, la troisième tombe sous les dispositions soit de la Loi sur les compagnies du Québec, c. 271, soit la Loi régissant les sociétés commerciales canadiennes, c. 33, malgré les articles 1889 à 1891 du Code.

Avant d'expliquer plus en détail les particularités de ces différentes formes de sociétés, il est utile de faire ici un rappel historique des lois dont l'influence

52. Voir l'art. 29 L.C.Q.

53. Voir aussi, au même effet, l'art. 61 du C.P.C.

54. André Couture, $D$ e la définition juridique du terme \& compagnie » en droit québécois, Québec, étude non publiée, faite pour le compte de l'Office de la langue française, 1973, p. 11-12.

55. Office de révision du Code civil, Rapport sur le Code civil du Québec, Québec, Editeur officiel, 1977 , vol. 1, p. 48-51.

56. Code civil de la province de Québec, Montréal, Wilson \& Lafleur, 1970, art. 1864. Nous traitons de la société anonyme plus loin dans la. présente étude. 
sur la création fort originale du système québécois a été importante. Il s'agit avant tout de lois anglaises; des lois françaises y figurent également pour donner à l'étude une dimension chronologique, bien qu'elles n'aient exercé qu'une influence négligeable sur les sociétés par actions au Québec.

Arunée Pays Lois

1844 Angleterre The Joint-Stock Companies Act

1850 Canada Acte pour autoriser la formation de compagnies à Fonds social pour la construction de chemins et autres travaux dans le Haut-Canada

N.B. Cette loi est indicative de toute une série de lois qui vont suivre, où il est question de préciser le fonctionnement des * compagnies à fonds social »; le tout conduira à la loi générale de 1861 .

1855 Angleterre The Limited Liability Act

$1856 \quad$ France Loi du 17 juillet sur les Sociétés en commandite par actions

Angleterre The Joint-Stock Companies Act

1861 Canada Acte relatif à l'incorporation judiciaires des compagnies à fonds social

1862 Angleterre The Companies Act

1863 France Loi du 23 mai 1863 créant la Société à responsabilité limitée

1867 France Loi du 24 juillet sur les sociétés commerciales

1868 Québec Actes des Clauses générales des Compagnies à fonds social (31, Vict., c. 24)

Acte concernant l'incorporation des compagnies à fonds social (31, Vict., c. 25)

Voilà donc une effervescence législative qui est assez singulière. Ce sont sans doute les lois anglaises, d'abord de 1844 et ensuite de 1856, qui ont eu la plus grande influence sur la législation canadienne. Comme nous l'avons vu, ces lois ont eu le même succès auprès des Français. $\AA$ cette époque, l'Angleterre éclairait la voie et les autres pays suivaient.

Dans les lois de 1868, il semble bien qu'au Québec, où les affaires se trouvaient principalement aux mains des anglophones, l'on ait copié de près le modèle canadien et la « Companies Act \$ anglaise de 1862. Le Québec s'est ainsi engagé dans une voie sensiblement différente de celle des Français.

L'adoption de \& l'Acte concernant l'incorporation des compagnies à fonds social \& n'a pu se faire sans sacrifier une orientation civiliste en matière com- 
merciale ; or, de toute manière, les juristes du Bas-Canada avaient déjà depuis longtemps axé le développement économique et juridique sur l'exploitation des modèles anglo-américains... La France, de par son retard dans le domaine, n'avait que peu à offrir ${ }^{57}$.

Telles sont les circonstances dans lesquelles les codificateurs québécois ont accepté trois formes de sociétés civilistes :

- la société en nom collectif

- la société en commandite

- la société anonyme.

Quant à la quatrième forme, ils durent innover. La France n'avait à proposer qu'un modèle transitoire, peu satisfaisant : la «société à responsabilité limitée », pauvre ancêtre de la société anonyme de 1867. Au Québec, les codificateurs ont opté pour l'appellation française de "société par actions», qualificatif que les Français appliqueront peu à leur classification des sociétés commerciales avant 1966. bécoises.

Voyons maintenant plus en détail les différentes formes de sociétés qué-

\section{La société en nom collectif}

La société en nom collectif est celle qui nécessite le moins de commentaires sur les plans linguistique et juridique, tant elle demeure semblable aux sociétés qui portent le même nom en France, en Belgique ou en Allemagne. Il faut signaler, cependant, quelques détails qui distinguent la variante québécoise.

À l'encontre de la société en nom collectif d’Europe, la société québécoise ne jouit pas de la personnalité morale, qualité réservée aux entités reconnues par l'État. Nous avons déjà constaté de quelle manière cette simple différence crée une divergence fondamentale entre le système civiliste du Québec et ceux d'ailleurs. Quant aux autres caractéristiques, retenons que la société québécoise est «enregistrée » auprès du gouvernement, et non «inscrite», que sa raison sociale ne fait pas usage du mot «compagnie » ou de l'abréviation « Cie», et enfin, qu'elle est sans doute la forme d'entreprise la plus répandue dans tout le Québec.

\section{La société en commandite}

Les articles 1871 à 1888 du Code civil traitent de la société en commandite et l'on n'y fait pas la distinction entre société simple et société par actions; à la lecture de ces articles, on comprend qu'il s'agit uniquement de la forme simple. Il est intéressant de connaître les origines de cette société du droit qué-

57. "In Lower Canada, the basis of private law was in theory \& La Coutume de Paris *, but in practice this jurisprudential system of law was strongly influenced by Common law. In the field of commercial law in particular, the courts apparently motivated by a desire for a degree of uniformity in commercial exchanges within North America, borrowed common law concepts, without much hesitation. ? D'après James Smith, op. cit., p. 26. 
bécois, car bien qu'elle dérive de loin d'un ancêtre du Moyen Âge, sa présence au. Québec s'est accomplie par une évolution sinueuse.

La société en commandite fait son apparition en Amérique du Nord en 1808 dans le «Digeste des lois civiles du territoire d'Orléans », c'est-à-dire dans le Code civil louisianais. De là, sa popularité se répand d'État en État et finit par atteindre le Haut-Canada ; la loi du Bas-Canada ne fit que calquer celle du Haut-Canada ${ }^{\text {ss. }}$.

Au cours de la première moitié du $\mathrm{xx}^{\ominus}$ siècle, cette société a été en perte de vitesse ; aujourd'hui, elle connaît un regain de vigueur, mais dans des circonstances imprévisibles : de très grandes entreprises s'en servent pour unir leurs capitaux afin de réaliser des ouvres communes, plutôt que de recourir à la création d'une compagnie par actions.

Enfin, tout comme la société en nom collectif, elle n'a pas la qualité de personne morale.

\section{La société par actions}

Des commentaires précédents sur la nature de la société par actions, il ressort que dès l'analyse, le masque francophone tombe et l'on est en présence d'une réalité anglo-saxonne : "the incorporated joint-stock company 》. Les nombreuses traductions de ce terme en français proviennent d'efforts vaillants chez les Canadiens francophones pour donner à cette institution un nom français précis. Nous avons constaté à quel point, pour des raisons historiques, ils n'ont pas eu la tâche facile.

Examinons un peu ces traductions, elles ont beaucoup à nous apprendre.

1. La société par actions (non) incorporée. - Dans cette expression, la présence anglaise est illustrée d'une façon particulière car à l'époque de la rédaction du Code civil, les codificateurs se sont référés exclusivement aux auteurs anglais, qui distinguaient entre les « unincorporated joint-stock companies » et les « incorporated joint-stock companies ». Malheureusement, le Québec n'a jamais connu cette distinction. Donc elle est, sur le plan juridique et linguistique, superflue, même incorrecte.

2. La compagnie par actions. - Cette expression ne semble refléter autre chose qu'une substitution du mot compagnie au mot société. Pour le reste, c'est un emploi particulier au Canada, difficilement exportable lorsqu'on considère sa résonance étrange pour des oreilles francophones non canadiennes.

3. La compagnie à fonds social. - C'est la traduction utilisée dans le titre de la section VII de la. Loi sur les compagnies; et pour cause, car ce très vieux terme remonte dans la législation québécoise et canadienne jusqu'aux lois des années 1840 .

Par ailleurs, l'expression « fonds social $\gg$ (C.C. 1873 et 1875) partage aussi le terrain avec \& fonds commun (C.C. 1872) et \&onds capital " (C.C. 1882).

58. André Couture, op. cit., p. 14. 
L'idée d'un «fonds commun » à la base d'une société remonte dans l'histoire au concept médiéval selon lequel les marchands s'associaient pour exploiter un commerce et, de ce fait, cotisaient à même leurs profits pour constituer un fonds commun, ce qui fut la naissance du joint-stock ${ }^{59}$.

$\mathrm{Au}$ Québec, certains praticiens, conscients de cette nuance, objectèrent à l'appellation «par actions », laquelle, selon eux, ne décrivait pas un aspect important de la compagnie québécoise. Le résultat en fut une étiquette que les puristes ne peuvent comprendre, car elle ne figure pas dans les dictionnaires de langue.

On trouve parfois aussi « compagnie à capital-actions». Le terme «capitalactions » présente encore un autre éclairage de ce qui entre dans la constitution d'une compagnie, c'est-à-dire l'apport en capital fourni par l'émission d'actions. Certes, l'expression est condamnée par ceux qui jettent l'opprobre sur tout ce qui ne figure pas dans les dictionnaires; toutefois, dans une compagnie où l'on veut éviter toute référence au concept de la société, que fait-on ?

4. La compagnie statutaire. - Il s'agit d'une compagnie constituée en vertu de la deuxième partie de la loi québécoise sur les compagnies, c'est-à-dire celle qui traite des joint-stock companies.

5. La compagnie publique (par opposition à la compagnie privée). - C'est la même distinction que l'on a vue plus haut à l'endroit de la société «onverte ou fermée», des open or closed (closely-held) corporations. Une explication précise figure dans la Loi modifiant la loi des valeurs mobilières, L.Q., 1971, chapitre 77 , article 1 .

Enfin, une dernière distinction du droit québécois qu'il convient d'établir provient de la personnalité morale d'une compagnie, car selon le juge Hall:

Every company is, of course, a corporation although every corporation is not a company ${ }^{60}$.

\section{La société anonyme}

Outre les trois catégories de société vues plus haut, il en existe également une quatrième dans le Code civil, la société anonyme. Cette forme de société possède les mêmes caractéristiqques que la société en nom collectif, à une exception près : elle ne porte ni de nom ni de raison sociale. Sa présence en droit québécois contemporain semble être un anachronisme, et le nouveau projet de Code civil ne parle plus de la société anonyme. Ailleurs, en droit civil moderne, l'usage veut que l'on appelle ce genre de société une association ou bien une société en participation.

Or, en 1864, les codificateurs québécois n'acceptèrent pas cette évolution juridique et linguistique : ils ont préféré ainsi garder l'ancienne formule.

La difficulté que présente cette tournure devenue archaïque provient de l'essor qu'a pris le terme rajeuni de "société anonyme». Donc, même si nous

59. L.C.B. Gower, Principles of Modern Company Law, London, Stevens, 1969, p. 29 et suiv.

60. Paroisse de Saint-Gabriel de Brandon c. Sarrazin (1953), 58 B.R., 123, 133. 
croyons que les codificateurs avaient vu juste, il vaudrait mieux qu'on abandonnât cet usage vieilli au Québec.

\section{Le projet de Code civil}

Lorsque le projet de Code civil aura franchi l'étape parlementaire et sera devenu une loi, on pourra sans doute constater la disparition de deux formes de société, et le changement de nom d'une troisième. En effet, le projet de code ne parle que des sociétés et de la commandite ${ }^{81}$, il n'y est plus question des sociétés anonymes ou par actions. La raison de ce nouvel état de choses est fort simple : dans le domaine des sociétés commerciales, depuis longtemps, le Québec a besoin d'une refonte complète de sa législation. Dans l'annexe 3, nous traitons du défi que ce changement comporte pour la terminologie juridique.

Ce nouveau code provoquera vraisemblablement une évolution plus civiliste du concept de société à cause d'une disposition succincte, mais lourde de

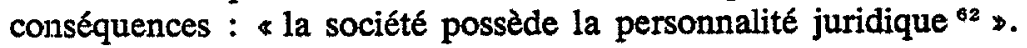

\section{Dualité de systèmes}

Selon que nous avons traité des questions relevant du droit civil ou du Common law, nous avons appliqué tout au long du présent exposé une dualité de méthodes. Elle est d'autant plus apparente lorsqu'on considère d'une part l'aisance avec laquelle il a été possible de classer les institutions civilistes et, d'autre part, les paramètres flottants qu'il a fallu créer pour décrire les entités du Common law. Ces démarches soulignent de nouveau comment le droit civil possède à la fois une structure externe et interne, tandis que le Common law n'a qu'une structure externe.

Si l'on compare les deux systèmes, on note pour l'un comme pour l'autre des avantages et des inconvénients ; un petit exemple suffit à le démontrer.

Le fait qu'une personne morale puisse relever, selon les circonstances, du droit public ou du droit privé vient compromettre l'étanchéité de ces catégories, ce qui incite souvent le civiliste à pousser à l'absurde un raisonnement binaire. Toutefois, le magma de structures possibles en droit anglo-américain conduit au désespoir et fait rêver au pays de cocagne où * tout n'est qu'ordre et beauté, luxe, calme et... \$ Revenons à l'entreprise.

\section{CONCLUSIONS}

Il est nombre de considérations d'ordre général que l'on peut tirer de notre exposé. La première, c'est la nature hybride du droit québécois, qualité qui ne lui a attiré, la plupart du temps, que de l'ingratitude à la fois des anglophones et des francophones ignorants des richesses de son histoire. Bien entendu, être entre le marteau et l'enclume est une situation qui ne plaît à personne ! Mais

61. Office de révision du Code civil, Rapport sur le Code civil du Québec, Québec, Editeur officiel du Québec, 1977 , p. $463,488$.

62. Ibid., p. 458. 
vouloir s'inspirer continuellement de deux grands systèmes juridiques ne peut guère conduire ailleurs.

En deuxième lieu, il ne faut point s'étonner que l'usage de l'anglais ou du français au Québec présente ses particularités. C'est souvent parce qu'il y a eu de bonnes raisons. Si défaut il y a, attribuons-le à l'évolution et à la réforme du droit québécois, parfois trop rapides au goût de l'opinion publique, et aussi aux gens de loi qui n'ont pas toujours suffisamment expliqué aux justiciables le bienfondé de certains changements.

Et en dernier lieu, après tout, ce n'est pas le fait d'être différent qui rend un peuple marginal ou inférieur, c'est plutôt de ne pas savoir pourquoi il est différent.

Wallace Schwab

ANNEXE 1

CANADA

Loi fédéralè

Québec

CODE CIVIL :

Société en nom collectif \% general partnership

\section{CORPORATION PUBLIQUE}

(corporation ou société de la couronne ou d'État)

\section{CORPORATION PRIVEE}

Société en commandite / partnership en commandite ou limited partnership

Société par actions / joint-stock company

Société anonyme / anonymours partnership

LOI SUR LES COMPAGNIES, ch. 271
FRANCE

SOCIETE EN NOM COLLECTIF

SOCIETE EN COMMANDITE SIMPLE

SOCIÉTÉ A RESPONSABILITÉ LIMITEE SOCIÉTE PAR ACTIONS

- société anonyme

- société en commandite par actions
ANGLETERRE

GENERAL PARTNERSHIP

LIMITED PARTNERSHIP

LIMITED LIABILITY COM-

PANY

(incorporated joint-stock company) 


\section{ANNEXE 2}

Le droit civil est à peu de chose près synonyme de droit des pays de langue latine s en ce sens que l'on trouve souvent dans les nations d'expression espagnole, italienne ou portugaise, un corps législatif qui a pris pour modèle le droit civil. Les quelques extraits du * Codigo de Sociedades Mercantiles y Cooperativas ${ }^{63}$ suffiront pour démontrer jusqu'à quel point la pensée juridique et, par extension, la structure même de ce droit s'approchent ou s'éloignent du modèle civiliste.

Artículo 1. Esta ley reconore las siguientes especies de sociedades mercantiles:

I. Sociedad en nombre colectivo;

II. Sociedad en comandita simple;

III. Sociedad de responsabilidad limitada ;

IV. Sociedad anónima :

V. Sociedad en comandita por acciones ; ...

Artículo 25. Sociedad en nombre colectivo es aquella que existe bajo una razón social y en la que todos los socios responden, de modo subsidiario, ilimitada y solidariamente de las obligaciones sociales.

Artículo 51. "Sociedad en comandita simple es la que existe bajo una razón social y se compone de uno o varios socios comanditos que responden, de manera subsidiaria, ilimitada y solidariamente, de las obligaciones sociales, $y$ de uno o varios comanditarios que ûnicamente estấn obligados al pago de sus aportaciones.

Artículo 58. Sociedad de responsabilidad limitada es la que se constituye entre socios que solamente estấn obligados al pago de sus aportaciones, sin que las partes sociales puedan estar representadas por títulos negociables a ta orden o al portador, pues sólo serán cedibles en los casos y con los requisitos que establece la presente ley.

Articulo 87. Sociedad anônima es la que existe bajo una denominación y so compone exclusivamente de socios cuya obligación se limita al pago de sus obligaciones.

Artículo 207. Sociedad en comandita por acciones es la que se compone de uno o varios socios comanditos que responden de manera subsdiaria, ilimitada y solidariamente, de las obligaciones sociales, y de uno o varios comanditarios que únicamente están obligados al pago de sus acciones.

63. Sociedades mercantiles y cooperativas, Mexico, Porrua, 1975, p. 25, 32, 36, 37, 43 . et 69 . 


\section{ANNEXE 3}

Vocabulaire bilingue des entreprises commerciales

AVERTISSEMENT : le moment n'est pas encore arrivé où traducteurs et juristes peuvent s'orienter à l'aide d'un vocabulaire «normalisé » des usages relevant du domaine des entreprises commerciales. À l'heure actuelle, la tâche des uns et des autres se révèle d'autant plus ardue que le choix d'un terme, au détriment d'un autre, reflète une orientation juridique et parfois une option politique particulières. Que nous soyons traducteur ou juriste, il est toujours gênant de constater qu'un mauvais choix pourrait engager notre responsabilité civile.

C'est ainsi que le vocabulaire suivant est descriptif, reflétant donc, pour le meilleur ou pour le pire, l'état de la règle de droit et celui de la langue. L'absence de consignes normatives et la présence de tournures bannies du bon usage ne manqueront pas de déplaire aux esprits purs.

Toutefois, ce n'est pas dans une typologie des structures commerciales qu'il convient de faire cuvre de réformateur. Une véritable épuration de ce vocabulaire ne résultera que d'une étroite collaboration entre juristes et terminologues. À ce propos, au Québec, une occasion en or risque de se présenter au cours de ces prochaines années. En effet, depuis bien longtemps, les juristes québécois reconnaissent la nécessité de refondre et de mettre à jour les nombreuses lois qui traitent des entreprises commerciales. La difficulté qui freine toujours les efforts les plus vaillants provient de l'ampleur de cette tâche. Aucun des gouvernements des quinze dernières années n'a pu mettre en marche une réforme de fond en comble de ces lois, et pour cause : une étude préparée par feu le notaire Yves Caron avait démontré l'immense complexité d'un tel travail. Ce n'est pas à un juriste, mais à une équipe de spécialistes qu'il faudra le confier.

Quoi qu'il en soit, ce jour de réforme n'est plus très loin.

Anonymous partnership

Appraiser

Articles of association

Assets (of a company).

Auditor

Beneficial owner

Beneficial ownership

Body corporate and politic

Business combination

Business name (cf. firm name and corporate name)

By-laws

Capital stock

Certificate of continuance
Société anonyme

(Qué.)

Association ou société en participation (Fr.)

Estimateur

Statuts

Patrimoine d'une société

Vérificateur

Véritable propriétaire

(Can.)

Propriété effective

(Can.)

Personne morale

Regroupement d'entreprises

(Can.)

Nom commercial

Règlements

Capital social ; capital-actions

Certificat de prorogation
(Can.)

(Can.) 
Certificate of incorporation

Charter

Civil liability

Closed corporation

Closely-held corporation

Consideration

Construction

Constructive delivery

Constructive ownership

Continuance

Corporate body

Corporate existence

Corporate name

Corporate records

Corporate seal

Corporate seat

Corporate signature

Corporate veil

Corporation

Corporator

Crown corporation

Cumulative voting

Custody

Dissolution

Distributing corporation

Dormant partner

Fiduciary

Firm name

General partner

General partnership

Guarantor

In.corporate (to)

Incorporated -ou- INC.
Certificat d'incorporation

(Can.)

Charte

Responsabilité civile

Compagnie privée

(Qué.)

Société fermée

(Fr.)

Compagnie privée

(Qué.)

Société fermée

(Fr.)

Contrepartie

(Can.)

Présomption

(Can.)

Présomption de livraison

(Can.)

Présomption de propriété

(Can.)

Prorogation

Personne morale

(Can.)

Existence d'un corps constitué; d'une société

Dénomination sociale

Registre de la corporation, de la société

Sceau de la compagnie

Siège social

Signature sociale

Paravent de la société

Corporation, corps constitué, personne morale

Fondateur (d'une société)

Corporation de la Couronne, société de la Couronne, société d'État

Vote cumulatif

Garde

(Can.)

Dissolution

Société ayant fait appel au public

Commanditaire

Représentant

Raison sociale

(Can.)

(Can.)

Associé

Société en nom collectif

Caution

(Belgique, France et Qué.)

Constituer en association, en compagnie, en corporation, en société; etc..; incorporer

Incorporée -ou- Inc. $\quad \because$ (Qué. et Can.)

Limitée -ou- Ltée (Qué. et Can.)

Société à responsabilité limitée -ou- S.A.R.I.

(Fr.) 


\section{Incorporator \\ Insider \\ Joint-stock company (incorporated)}

Joint-stock company (unincorporated)

Legal person

Legal representative

Letters patent

Liability

Lien

Limited liability

Limited partnership

Memorandum of association

Natural person

Offer

Offeror

Open corporation (U.S.)

Partner

Proxy

Proxy holder

Public corporation

(cf. Crown corporation)
Receiver

Recovery

Redemption (of shares)

Registered -ou- Reg'd

Registered office

Registrant .

Registration
Société de personnes à responsabilité limitée -ou- S.p.A.R.L. ou Société anonyme, S.A.

Fondateur

Initié

(Belgique)

(Can.)

Société par actions

(Qué.)

Compagnie par actions

(Qué.)

Compagnie à fonds social

(Qué.)

Société à responsabilité limitée ou anonyme

Société de personnes à responsabilité limitée - ou société anonyme

(Belg.)

Société en nom collectif

(Qué. et Fr.)

Personne morale

Mandataire

(Can.)

Lettres patentes

Créance - dette - obligation - passif

Privilège

Responsabilité limitée

Société en commandite (simple)

Statuts

Personne physique

Pollicitation

(Can.)

Pollicitant

(Can.)

Compagnie publique

(Qué.)

Société ouverte

(Fr.)

Associé

Procuration

(Can.)

Fondé de pouvoir

(Can.)

Corporation publique

Séquestre

Recouvrement

Rachat (des actions)

Enregistré -ou- Enr.

Et Cie

Siège social

Courtier attitré

Enregistrement

Inscription / Immatriculation
(Can.)

(Can.)

(Qué.)

(Fr.)

(Can.)

(Can.)

(Qué.)

(Fr.) 
Representative action

Scrip certificate

Security certificate

Share

Shareholder

Shareholder agreement

Short sales

Sleeping partner

Society

Stated capital

Stock

Stockholder

Take-over bid

Trust indenture

Trustee

Waiver

Wind up (to)

Winding up
Action en justice collective

(Can.)

Recours collectif

(n'existe pas en dr. franç.)

Scrip

Certificat de valeur mobilière

Action:

Actionnaire

Convention des actionnaires

Ventes à découvert

Commanditaire

Société

Capital déclaré

Action

Actionnaire

Offre d'achat visant à la mainmise

(Can.)

Acte de fiducie

Fiduciaire

(Can.)

(Can.)

(Qué.)

(Can.)

(Que.s)

Renonciation

Liquider

Liquidation

BIBLIOGRAPHIE

AMOS, Sir M.S. et F.P. WALTON, Introduction to French Law, 2e Ed, Oxford, Clarendon, $1963,398 \mathrm{p}$.

BRELINGARD, Désiré, et al., Histoire, le monde de 1715 a 1870, Paris, Hachette, 1966; $320 \mathrm{p}$.

CODE CIVIL de la province de Quebec, $22^{\circ}$ Edition bilingue, Montreal, Wilson \& Lafleur, $1970,782 \mathrm{p}$.

CODE DE COMMERCE, coll. Petits codes, Paris, Dalloz, 1977-1978, 1208 p.

CODE DE PROCÉDURE CIVILE de la province de Québec, (1965) Lois du Québec, c. 80, Don Mills, Ont., Kingsiand Publications, 1978, 598 p.

COUTURE, André, $\mathrm{De}$ la définition juridique du terme ecompagnie.s en droit québécois, Québec, étude non publiée, faite pour le compte de l'Office de la langue française, 1973.

DIDIER, Paul, Droit commercial, coll. Thémis, Paris, P.U.F., 1970, 746 p.

DIDIER, Paul, Droit commercial, les entreprises, coll. Thémis, Paris, P.U.F., 1972, 538 p.

DRUCKER, Peter F., The Concept of the Corporation, 4 éd., New York, Mentor; 1964, $255 \mathrm{p}$.

ENCYCLOPADIA BRITANNICA : Macropaedia, ed.. : William Benton et Helen H: Benton, Toronto, Encyclopaedia Britannica Inc., 1975, 30 vol.; et Ed. W. Benton, Toronto, Britannica, 1971, 23 vol.

ENCYCLOPAEDIA UNIVERSALIS, publiée sous le haut patronnage de R. Aron et al, Paris, Encyclopaedia Universalis France S.A., 1973, vol: 15:" 
FRANKLIN, Martin et David R. FRANKLIN, Introduction to Québec Law, Toronto, Sir Isaac Pitman Ltd., 1972, 327.p.

GIFIS, Steven H., Law Dictionary, Woodbury (N.Y.), Barrow's Educational Series, 1975, 227 p. GOWER, L.C.B., Principles of Modern Company Law, 3e éd., London, Stevens, 1967.

GOWER, L.C.B., \& Some Contrasts between British and American Corporation Law », in Harvard Law Review; 1969, p. 1371-1372.

GUILLIEN, Raymond et Jean VINCENT, Lexique de termes juridiques, Paris, Dalloz, 1972, $353 \mathrm{p}$.

HOGHTON, Charles de, The Company law, structure and reform in eleven countries; London, Allen \& Unwin Ltd., 1970, 394 p.

JUGLART, Michel de, et al., Leçons de droit civil, les personnes, tome premier, se éd., Paris, Éditions Montchrestien, 1972, 714 p.

LEPOINTE, G., Petit vocabulaire d'histoire du droit français, Paris, Domat Montchrestien, 1948,390 p.

LEXIS, Dictionnaire de la langue française, dir.: Jean Dubois, Paris, Larousse, 1975, $1950 \mathrm{p}$.

Loi des Compagnies, L.Q. 1964 et modifications suivantes, Québec, Imprimeur de la Reine et Éditeur officiel du Québec.

Loi régissant les sociétés commerciales canadiennes, (c. 33, 23-24 Elizabeth In), Farnham, Qué., Éditions FM Compilations, 1977.

Loi sur la corporation commerciale canadienne, S.R. c. 35, Farnham, Québec, Éditions FM Compilations, 1978, 6 p.

Loi sur la radiodiffusion 1967-1968, c. 25, Farnham, Québec, Editions FM Compilations, 1976, 27 p.

Louisiana Civil Code, éd. Joseph Dainow, St. Paul, Minn., West, 1972, Compiled Edition.

MUNN, Glen G., Encyclopaedia of Banking and Finance, éd. : F.L. Garcia, Boston, Bankers Publishing Co., 1962, 788 p.

NICOLAS, Barry, An Introduction to Roman Law, Oxford, Clarendon Press, 1962, 281 p.

Office de Révision du Code Civil, Rapport sur le Code civil du Québec, vol. 1, projet de Code civil, Québec, Editeur officiel du Québec, 1977, 786 p.

OSBORN, P.G., A Concise Law Dictionary, se éd., London, Sweet \& Maxwell, 1964, 394 p.

PALMER, R:R., A History of the Modern World, New York, Knopf, 1960, 945 p.

PEYRET, Henri, la Stratégie des trusts, Paris, P.U.F., coll. «Que sais-je ?», 1966, 128 p.

Régie de la langue française, Comment formuler une raison sociale en français, Québec, Editeur officiel du Québec, 1976, 31 p.

RICHERT, John P. et E. Suzanne RICHERT, The Impact of the Civil Code of Louisiana upon the Civil Code of Québec 1866 ;, in Thémis (1973), 8 R.J.T,, no 3, p. 501-520.

RODIÈRE, René et Roger HOUIN, Précis de droit commercial, tome 1, Paris, Dalloz, 1970.

RYAN, K.W., An Introduction to the Civil Law, Toronto, Carswell, 1962, 294 p.

SCHWAB, Wallace, Lexique bilingue raisonné des lois de l'impôt sur le revenu au Canada, Montréal, thèse de maîtrise non publiée, 1972, $212 \mathrm{p}$.

SHEPPARD, Claude-Armand, The Law of languages in Canada, Ottawa, Information Canada, $1971,414 \mathrm{p}$.

SMITH, James, «The Representation of Companies in Québee \$, in Thémis (1973), 8 R.J.T., n'.1, p. 25-63.

Sociedades Mercantiles y Cooperativas, coll. Porrua, Leyes y Codigos de Mexico, 23 ed. Mexico, Editorial Porrua, S.A.; 1975, 228 p.

VILLEY, Michel, le Droit romain, Paris, P.U.F., coll. « Que sais-je ? \$; 1972, 125 p.

Webster's New World Dictionary, Second College Edition, éd. : David B. Guralnik, Toronto, Nelsón, Foster \& Scótt Ltod., 1970, 1692 p. 